\title{
Autonomous microfluidics with stimuli-responsive hydrogels
}

\author{
Liang Dong† and Hongrui Jiang* \\ Received 1st May 2007, Accepted 3rd August 2007 \\ First published as an Advance Article on the web 23rd August 2007 \\ DOI: $10.1039 / \mathrm{b} 706563 \mathrm{a}$
}

There has been increasing interest in integrated microfluidic systems because performing biological and chemical laboratory tasks on a single chip is appealing. One straightforward approach to constructing these 'lab on chips' is to fabricate individual components and to assemble them for desired functionalities. As the functionalities of the microfluidic systems become increasingly complicated, more functional components and relevant controls need to be integrated on a miniaturized chip, especially when a closed loop is needed for autonomous functionality. Instead, an emerging approach is to incorporate stimuli-responsive hydrogels directly into microfluidics to reduce the system complexity. Due to the hydrogels' ability of transducing stimuli into mechanical actions in response to their surrounding aqueous environment, hydrogel-based microfluidic elements can act as both sensors and actuators simultaneously, alleviating the requirement of most controls and even power sources. This provides microfluidic systems with autonomous functionalities. In this article, we will focus on a few autonomous microfluidic devices including valves, flow sorters, $\mathrm{pH}$ regulators, pumps, mixers, drug-delivery devices, fluidic cooling devices, and liquid microlenses.

\section{Introduction}

Microfluidics is the science and technology of designing and manufacturing devices that deal with the behavior, precise control and manipulation of small volumes $\left(10^{-9}-10^{-18}\right.$ litres $)$ of fluids. ${ }^{1}$ As a new research field that emerged in the 1990 s,

Department of Electrical and Computer Engineering, University of Wisconsin-Madison, 1415 Engineering Drive, Madison, WI 53706, USA.E-mail: hongrui@engr.wisc.edu

$\uparrow$ Present address: Department of Electrical and Computer Engineering, Iowa State University, 2115 Coover Hall, Ames, IA 50010, USA. microfluidics has shown great potential to revolutionize chemical synthesis and biological analysis by providing automation, high accuracy, low time and energy consumption, and great reconfigurability. ${ }^{1-4}$ These advantages have been demonstrated by recent progress in the development of a variety of 'lab on chips' or 'micro total analysis systems' for healthcare, ${ }^{5}$ cellomics, ${ }^{6}$ genomics, ${ }^{7}$ proteomics, ${ }^{7}$ and microoptics. ${ }^{8}$ Essentially, a 'lab on a chip' is a microfluidic device that has tiny channels and components to perform fluid handling; multiple components with different functionalities can be integrated onto the same chip. A functional microfluidic device may perform various biological or chemical

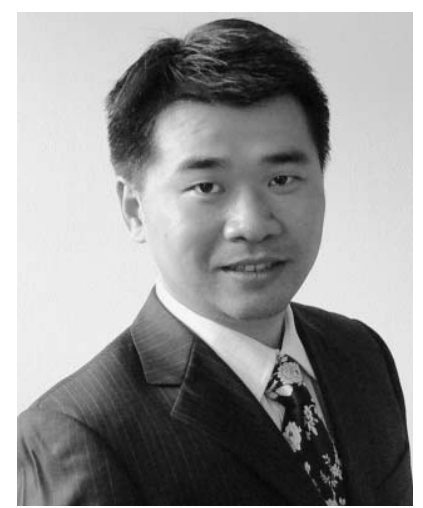

Liang Dong
Liang Dong received his B.S. degree in precision instrumentation from Xidian University, China, in 1999, and Ph.D. degree in electronics science and technology from Tsinghua University, Beijing, China, in 2004. From 2004 to 2007, he worked at the Department of Electrical and Computer Engineering, University of Wisconsin-Madison as a postdoctoral researcher. He is currently working at the Department of Electrical and Computer Engineering, Iowa State University as an assistant professor. He was the recipient of the National Outstanding Doctoral Dissertation Award of China in 2007. His research interests include BioMEMS, biosensors, microfluidics, lab on chips, smart materials, and biomimetics, and their applications in biomedical engineering and health care.

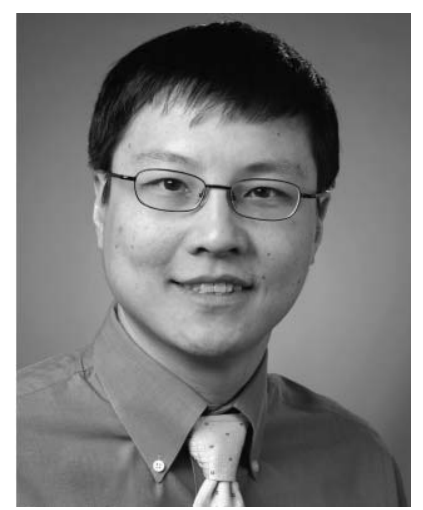

Hongrui Jiang received his B.S. degree in physics from Peking University, Beijing, China. He received M.S. and Ph.D. degrees in electrical engineering from Cornell University, Ithaca, NY, in 1999 and 2001, respectively. He is currently an assistant professor at the Department of Electrical and Computer Engineering, a faculty affiliate with the Department of Biomedical Engineering, and a faculty member of the Materials Science Program at University of Wisconsin-Madison. His research focuses on microfabrication technology, biological and chemical microsensors, microactuators, optical MEMS, smart materials and microlnano structures, and lab on chips. Before he joined the faculty of University of Wisconsin-Madison in 2002, he was a postdoctoral researcher at the Berkeley Sensor and Actuator Center, University of California, Berkeley, from 2001 to 2002. 
processing from sampling and pre-processing to sensing and measurement involved in an assay.

In general, microfluidic devices have planar structures with a scale similar to that of semiconductor integrated circuits. Therefore, they can be relatively integratable with electrical, mechanical, or optical elements, to realize functionally complex lab on chips. Significant progress has been made to realize a variety of sensors, such as biochemical, ${ }^{9}$ fluorescence, ${ }^{10}$ flow, ${ }^{11}$ temperature, ${ }^{12}$ and pressure $^{13}$ sensors. Other important elements, such as valves, ${ }^{14}$ pumps, ${ }^{15}$ mixers, ${ }^{16}$ filters, ${ }^{17}$ reactors, ${ }^{18}$ and heating and cooling elements ${ }^{19,20}$ have also been demonstrated. As the microfluidic systems become increasingly complicated to provide more functions, more functional elements need to be integrated on a miniaturized chip. However, this generally requires complicated control systems for the operation of these components. For instance, autonomous functionality needs to form a closed loop between each sensor and actuator through a control system, which would be challenging. Also, scaling down some of these components onto a single chip is nontrivial. If there is a way to combine the functionalities of sensing and actuation into a single component, it will simplify the control of the whole system and thus reduce the complexity.

\section{Using stimuli-responsive hydrogels for autonomous operation in microfluidics}

Beebe and his colleagues devised an interesting way to link sensing to actuation in $2000 .^{21}$ Stimuli-responsive hydrogels were integrated into microfluidics, acting as both sensors and actuators in these systems. These hydrogels have a significant advantage over conventional microfluidic actuators owing to their ability to undergo abrupt volumetric changes in response to their surrounding aqueous environment without the requirement of external controls and even power sources, ${ }^{22}$ thus providing microfluidic systems with autonomous functionality. The volumetric change renders a mechanical force and work, transducing a stimulus into a mechanical action, as in muscles. ${ }^{23}$ The factors affecting the force are the dimension of the hydrogel structure, the chemical composition of the polymer matrix, and the environmental conditions. ${ }^{24}$

Generally, stimuli-responsive hydrogels are a class of crosslinked polymers that have the ability to absorb water. They can undergo phase transitions, wherein an external stimulus, such as $\mathrm{pH},{ }^{25}$ glucose, ${ }^{26}$ antigen, ${ }^{27}$ protein, ${ }^{28}$ temperature, ${ }^{29}$ light, ${ }^{30}$ or electric field, ${ }^{31}$ gives rise to distinct volumetric changes in these hydrogels. Stimuli induce physico-chemical changes in the hydrogel polymer network. This then affects the movement of water and ions into and out of the polymer matrix. For instance, in the temperature-sensitive hydrogel poly( $N$-isopropylacrylamide), or poly(NIPAAM) (Fig. 1(a)), the movement of water is initiated by a change in the hydrophilicity of the backbone; in the $\mathrm{pH}$-sensitive hydrogel poly(2-hydroxyethyl methylacrylate-co-acrylic acid), or poly(HEMA-co-AA) (Fig. 1(b)), the movement of water is initiated by the ionization of the backbone. ${ }^{32}$ Since the movement of ions or molecules and water into the hydrogel is mainly by diffusion, the time scales for the volumetric change depend on the distance of diffusion or the size of the

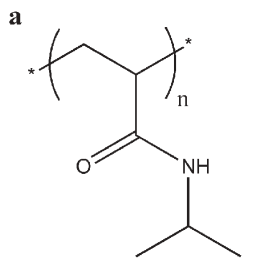

poly(N-isopropylacrylamide) poly(NIPAAM) b

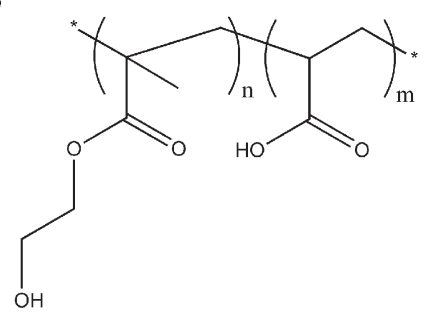

poly(2-hydroxyethyl methacrylate-co-Acrylic acid) poly(HEMA-co-AA)
Fig. 1 Chemical structures of two stimuli-responsive hydrogels as examples: (a) temperature-sensitive hydrogel poly(NIPAAM); and (b) pH-sensitive hydrogel poly(HEMA-co-AA).

hydrogel. ${ }^{21}$ Scaling down the size of hydrogel structures to micrometres can lead to a response time of a few seconds to subseconds. ${ }^{33}$ The hydrogel structures can be patterned using approaches similar to those for photoresists that are widely used in semiconductor industry. ${ }^{33-36}$ A thorough description of the chemical structures and the synthesis of various hydrogels is beyond the scope of this article, and the readers are directed to a few review papers for more information. ${ }^{37-40}$

Through the use of a variety of hydrogels, autonomous microfluidics can be realized. ${ }^{21,41}$ Here, we will review a few examples of autonomous microfluidic devices including valves, flow sorters, $\mathrm{pH}$ regulators, pumps, mixers, drug-delivery devices, fluidic cooling devices, and liquid microlenses.

Valves are one of the most crucial components for flow control in microfluidic systems. ${ }^{14}$ Their on/off state accomplishes regulation of fluid flow in channels. A conventional active valve consists of a deformable diaphragm coupled to an actuator that externally controls the diaphragm to open or close a channel. This approach is challenging to construct microfluidics because the diaphragm must be flexible and integratable with the actuator. ${ }^{32}$ So far, a number of hydrogel microvalves have been developed that are capable of autonomously making a decision and acting. ${ }^{21,34,42-46}$ A simple version of a hydrogel valve was designed by directly using hydrogel structures as the active control. ${ }^{21,42}$ For example, a smart channel was made by either patterning two strips of poly(HEMA-co-AA) $\mathrm{pH}$-sensitive hydrogel along the walls of a channel ${ }^{42}$ or an array of the same type of hydrogel posts in a channel (Fig. 2). ${ }^{21}$ The swelling of these hydrogel structures blocked a channel when a high $\mathrm{pH}$ buffer flowed into the channel. At a contracted state when $\mathrm{pH}$ was appropriately decreased, the fluid was allowed to pass the valve.

Based on the autonomous valve mechanism described above, a flow sorter that is able to divert a flow down different paths responding to the $\mathrm{pH}$ of the fluid has been realized (Fig. 2). ${ }^{21}$ The device sensed the $\mathrm{pH}$ of the input flow and routed the fluid down one of two paths based on the $\mathrm{pH}$ of the input. The poly(HEMA-co-AA) hydrogel in one branch expanded in high $\mathrm{pH}$ and contracted in low $\mathrm{pH}$, while a poly(HEMA-co-(dimethylamino)ethyl methacrylate), or poly(HEMA-co-DMAEMA) hydrogel gated the other branch and exhibited the reverse behavior. When high $\mathrm{pH}$ solution flowed into the device, the fluid flowed toward the contracted gel since the other channel was completely blocked at the expanded 

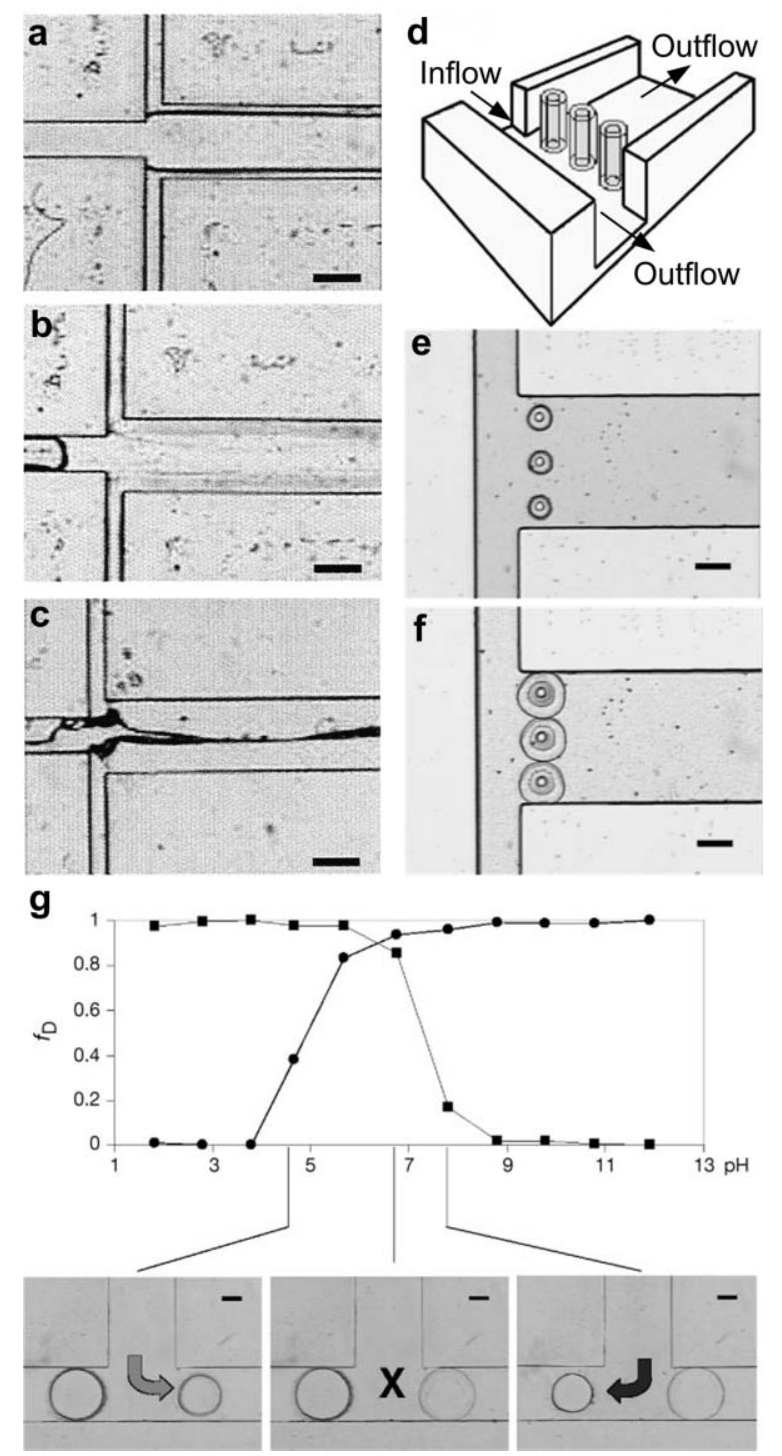

Fig. 2 Autonomous flow control in microfluidic channels using hydrogel valves. $(\mathrm{a}-\mathrm{c})$ A throttle valve fabricated using the 'laminar stream mode.' Scale bars represent $300 \mu \mathrm{m}$. (a) Three steady laminar streams are flowed in the channel. The outer streams consist of pre-polymer mixture of $\mathrm{pH}$-sensitive hydrogels. The middle stream consists of glycerin. (b) Hydrogel strips are photopolymerized along the channel walls, while glycerin is still flowing in the middle stream. (c) When exposed to a basic solution, the pH-sensitive hydrogel strips expand to seal off the channel. (d-f) Using a series of hydrogel posts as a valve. Scale bars represent $300 \mu \mathrm{m}$. (d) A diagram of the hydrogel jackets around the central posts. (e) The valve blocks the side channel branch in their expanded state at high $\mathrm{pH}$. (f) The contracted hydrogels at low $\mathrm{pH}$ allows fluid to flow down the side branch. (g) A flow sorter. Scale bars represent $300 \mu \mathrm{m}$. The plot on the top shows the fractional change in diameter $\left(f_{\mathrm{D}}\right)$ of the hydrogels with respect to $\mathrm{pH}$. At neutral $\mathrm{pH}$, the flow goes left and right; however, at high $\mathrm{pH}$ one hydrogel expands while the other contracts to direct fluid rightward. The opposite occurs at low $\mathrm{pH}-$ the black gel expands in high $\mathrm{pH}$ while the white gel expands at low $\mathrm{pH}$. (a-c) are reproduced from ref. 34. Illustrations $(\mathrm{d}-\mathrm{g})$ are reprinted by permission from Macmillan Publishers Ltd, Nature, ${ }^{21}$ copyright 2000 . state of the other hydrogel. Here, each hydrogel valve acted to sense, actuate and regulate the flow direction.

$\mathrm{Yu}$ et al. demonstrated a biomimetic check valve using $\mathrm{pH}-$ sensitive hydrogel poly(HEMA-co-AA) inside a glass microchannel (Fig. 3) ${ }^{34,43}$ Natural valves found in veins gave the inspiration for the design of this device; they are usually found in pairs, attached by their convex edges to the wall of the vein, which restricts the reflux of blood by bringing the opposing edges into contact. The fabricated valve activated and deactivated in response to solution $\mathrm{pH}$ due to the $\mathrm{pH}$ responsive hydrogel in the two strips of the valve. When exposed to high $\mathrm{pH}$, dissimilar expansion in adjacent hydrogel strips caused the valve to bend during swelling, forming a normally closed check valve that only allowed forward flow once the pressure reached a threshold value. At low $\mathrm{pH}$, the valve became deactivated due to the shrinking of the hydrogel, allowing both forward and backward flow.

Self-regulated microfluidic systems are important for chemical reactions and biological assays that are sensitive to environmental conditions, and hold promise for controlled drug delivery. Many microfluidic $\mathrm{pH}$ regulation schemes have been demonstrated. ${ }^{47,48}$ However, these conventional systems require several building block components (actuators, sensors and control systems). Using a poly(HEMA-co-AA) $\mathrm{pH}$ responsive hydrogel to replace these major components, an

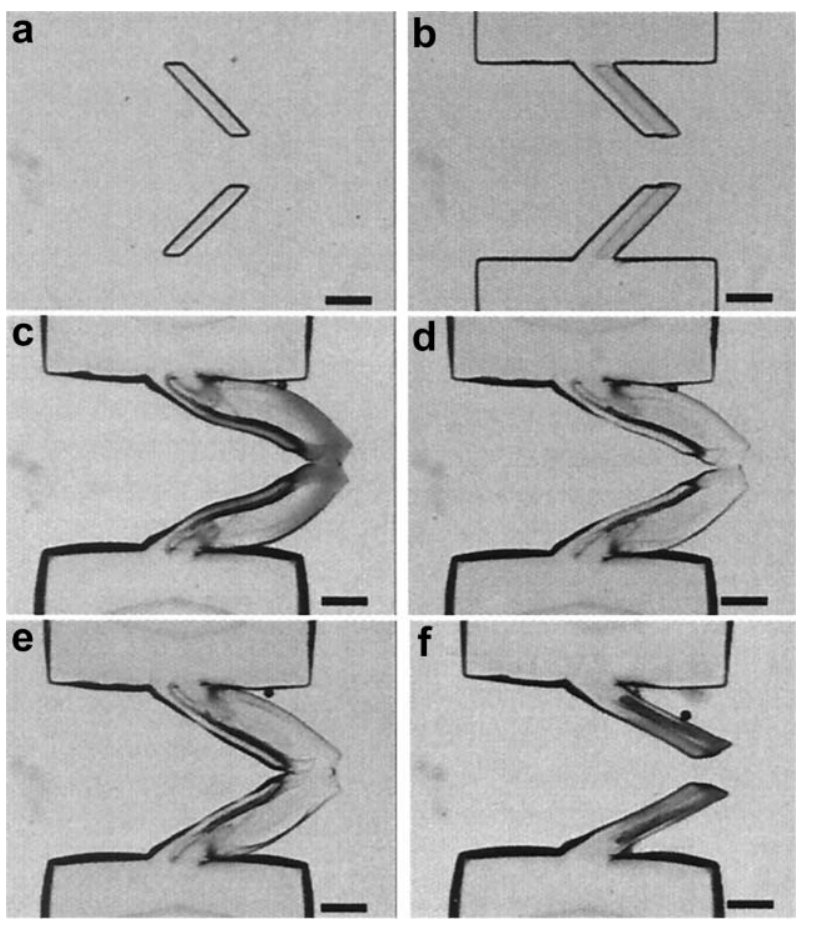

Fig. 3 Fabrication and operation of a biomimetic valve based on bistrip hydrogel. (a) Bistrip hydrogel is patterned by simultaneous photopolymerization. (b) The anchor of the valve is formed using a non-responsive hydrogel. (c) When exposed to basic solution, the bistrip hydrogels expand and curve to form a normally closed valve. (d) The bistrip valve is pushed open to allow flow in one direction (from left to right). (e) The flow is restricted in the opposite direction. (f) When exposed to acidic solutions, the valve is deactivated, returning to the permanently open state. Scale bars represent $500 \mu \mathrm{m}$. Images reproduced from ref. 34 . 
a

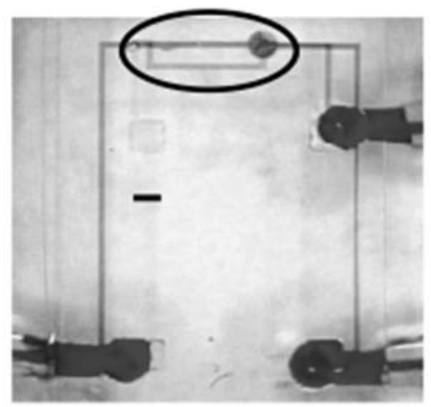

b

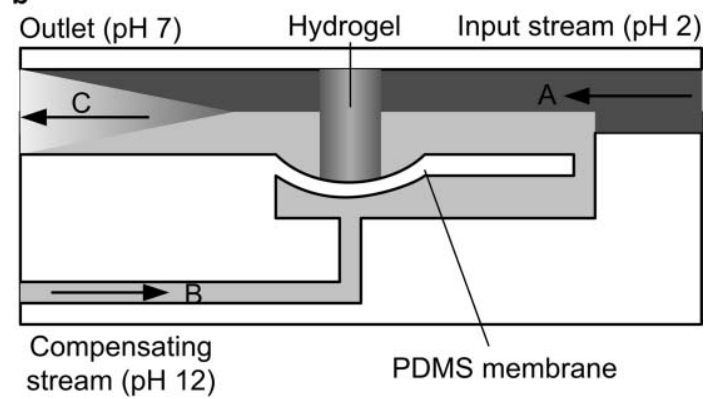

Fig. 4 A pH-regulation device. (a) Top view of the device. Scale bar represents $1 \mathrm{~mm}$. (b) Schematic of side view of the device to demonstrate the flow conditions. The compensating buffer enters from the bottom left (stream B, pH 12), while the input enters from the top right (stream A, pH 2). The two streams meet and then flow past the hydrogel post from right to left and exit the outlet on the top left (purple, $\mathrm{pH}$ 7). The regulated stream is initially separated, but the flow lengths are adequate to achieve complete diffusion between the two streams at the outlet. Images reproduced and adapted from ref. 49, 50, by permission of the Royal Society of Chemistry (copyright 2001) and Elsevier (copyright 2004).

interesting device that can continuously regulate an outlet stream to a desired $\mathrm{pH}$ value has been realized (Fig. 4). ${ }^{49,50}$ The hydrogel post was positioned inside the fluid being regulated, coupling the regulation of the fluid directly to the hydrogel volume; it sat on a flexible membrane that deflected down as the hydrogel expanded to occlude an orifice. Stream A represents the input flow $(\mathrm{pH} 2)$, stream $\mathrm{B}$ represents the compensating stream $(\mathrm{pH} 12)$, and stream $\mathrm{C}$ represents the regulated stream $(\mathrm{pH} 7)$. The system autonomously maintained a steady $\mathrm{pH}$ within a certain range of flow conditions. The $\mathrm{pH}$ to which the device regulates was mainly determined by the chemistry of the hydrogel sensor/actuator.

By taking advantages of autonomous flow control within microfluidic channels, autonomous microfluidic drug-delivery devices have been demonstrated. Conventionally, one needs control algorithms, circuitry, power, mechanical sensors, and pumps to control the concentration of the drug in the blood. A novel device similar to the $\mathrm{pH}$ regulation device described above used the volume expansion of a poly(methacrylic acidtriethylene glycol dimethacrylate), or poly(MAA-EG) $\mathrm{pH}$ responsive hydrogel disc to deform a membrane and to occlude an orifice preventing drug release (Fig. 5a). ${ }^{50,51}$ This hydrogel was loaded with two enzymes, glucose oxidase and catalase, to convert a glucose concentration change to a $\mathrm{pH}$ change. The volumetric change of the hydrogel was regulated by glucose concentration. The contraction of hydrogel could cause the
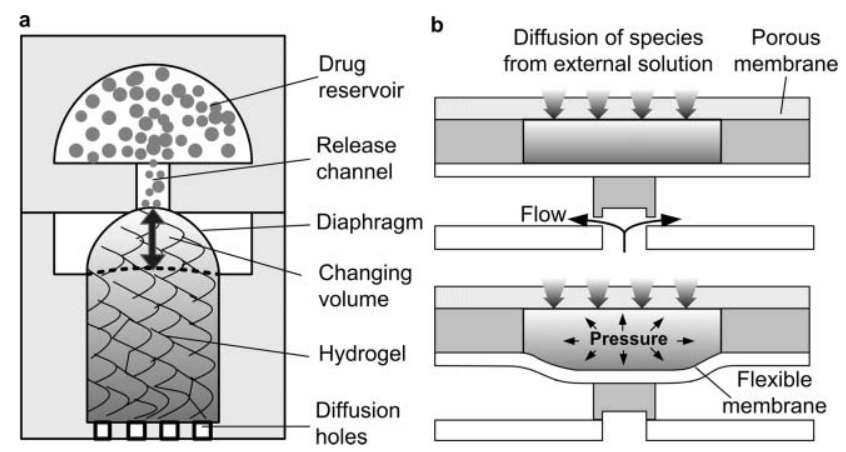

Fig. 5 Drug delivery using hydrogel. (a) A self-regulated drugdelivery device. Acrylic polymer is computer-numerical-controlmachined to make all the parts of the device. The hydrogel switch cavity was partially filled with hydrogel. One end of the release channel was connected to the central portion of the drug reservoir, while the other end of the channel can be either opened or blocked depending on the status of the pH-sensitive hydrogel in the switch cavity. Two enzymes, glucose oxidase and catalase, are used to convert the glucose concentration change to a $\mathrm{pH}$ change. (b) Hybrid MEMS-hydrogel drug-delivery device. Images reproduced and adapted from ref. 46, 51, with kind permission from Springer Science and Business Media (copyright 2001) and IEEE (copyright 2003).

orifice to open, thus diffusing drug out in a controlled manner. Since the hydrogel used here was synthesized with bulk polymerization techniques with a large dimension $(15 \mathrm{~mm}$ in diameter), the time needed for the device was a few hours. Ziaie and his colleagues improved the time performance by using micro-electro-mechanical-systems (MEMS) fabrication techniques (Fig. 5(b)) ${ }^{46}$ Specifically, they used a valve that constituted a smaller responsive hydrogel sandwiched between a rigid porous membrane and a flexible silicone-rubber membrane, thus decreasing the time response of the device.

In microfluidic systems, micromixers and micropumps are essential components for fluidic handling. Numerous designs of micromixers exist and help facilitate the development of microfluidic systems for mixing at the microscale. ${ }^{52-56}$ Likewise, a variety of micropumps have been developed based on different functioning methodologies. ${ }^{57-60}$ Many of these systems require on-chip power, costly and time-consuming fabrication processing, and/or experience difficulties integrating these systems with other platforms, such as biological systems. To improve on this, autonomous micromixers and micropumps have been developed using $\mathrm{pH}$ - and temperatureresponsive hydrogels, electroplated nickel $(\mathrm{Ni})$ impeller, and magnetic stirrers. ${ }^{61} \mathrm{~A}$ responsive hydrogel ring that behaved much like a clutch was patterned at the center axis of the $\mathrm{Ni}$ impeller (Fig. 6a). For example, consider a pH-sensitive poly(HEMA-co-AA) hydrogel ring that expands in basic solutions and shrinks in acidic solutions. The $\mathrm{Ni}$ impeller was coupled with an underlying rotating magnetic stirrer that was constantly on. When the local environment $\mathrm{pH}$ was decreased, the hydrogel ring shrank, allowing the $\mathrm{Ni}$ impeller to rotate freely; however, when the local $\mathrm{pH}$ was raised above its transition point, the hydrogel ring expanded into a mushroom cap to exert both downward and lateral forces on the $\mathrm{Ni}$ impeller, thus stopping the rotation of the $\mathrm{Ni}$ impeller. Fig. 6(b-d) shows pH-responsive micromixer and a mixer 

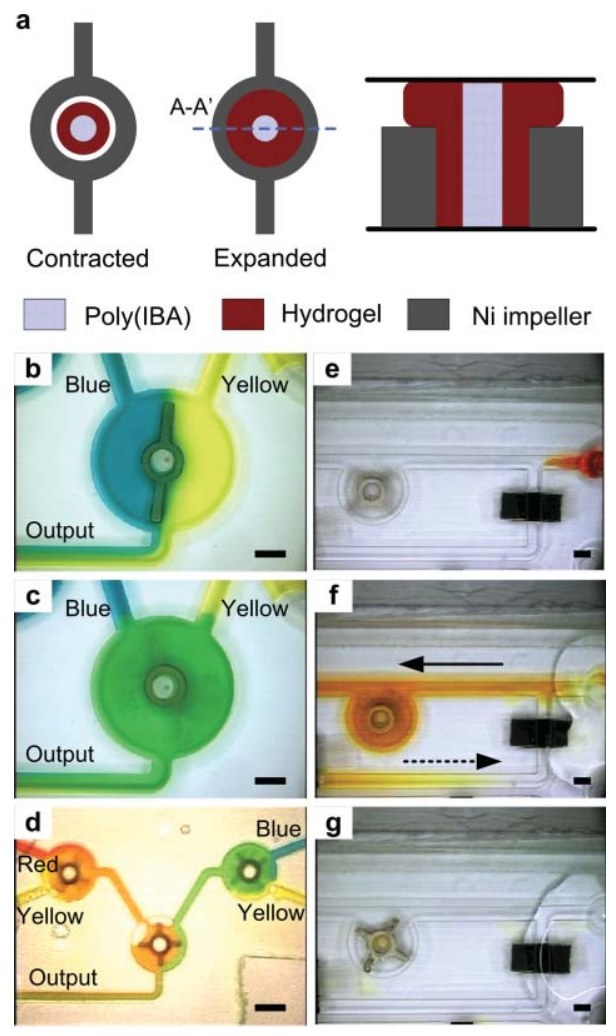

Fig. 6 Autonomous microfluidic mixers and pumps. (a) Conceptual diagram of using hydrogel to control the actuation of a Ni rotor, much like an automotive clutch. (b-d) A microfluidic mixer using a $\mathrm{pH}$ responsive hydrogel. Scale bars represent $1 \mathrm{~mm}$. (b) Low $\mathrm{pH}$ causes the hydrogel ring to shrink in volume, thus allowing mixing two flows in the chamber. (c) High $\mathrm{pH}$ causes the hydrogel ring to expand in volume, constricting the Ni rotor's rotational movement. (d) An array of three micromixers mixing dyed water solutions. (e-g) A microfluidic pump using temperature-sensitive hydrogel. The hydrogel axle expands and contracts, controlling the pumping action of the fluid. Scale bars represents $1.0 \mathrm{~mm}$. (e) Warm water circulates through the channels. A drop of yellow dye is placed at the input. (f) The dye has been pumped to the left on the top channel (shown by top dashed arrow) and recirculated back along the bottom channel to the right (shown by bottom dashed arrow). Cold water is placed at the input. (g) The micropump stops pumping once the hydrogel expands sufficiently. Images reproduced from ref. 61 (copyright 2005, IEEE).

array developed with a pH-sensitive poly(HEMA-co-AA) hydrogel ring at the center axis of each $\mathrm{Ni}$ impeller. When the stirrer was not activated, no mixing occurred despite the flowing of two adjacent liquid streams. Upon activation of the stirrer at low $\mathrm{pH}$ environment, the $\mathrm{Ni}$ impeller rotated and mixed the blue and yellow-dyed water solutions into a homogeneous green solution. Autonomous temperature-sensitive micropumps, based on the concept of recirculation, which autonomously decided when to pump fluid, were also developed (Fig. 6(e-g)). Because of the rotating actuation of the $\mathrm{Ni}$ impeller, a change in pressure was created within the microchannels, thus creating the driving force necessary to pump the liquid. Poly(HEMA-co-DMAEMA) hydrogel was used in this device. It was reported that a rotating impeller could effectively stop pumping as temperature decreased, while higher temperatures called the pumping into service. ${ }^{61}$

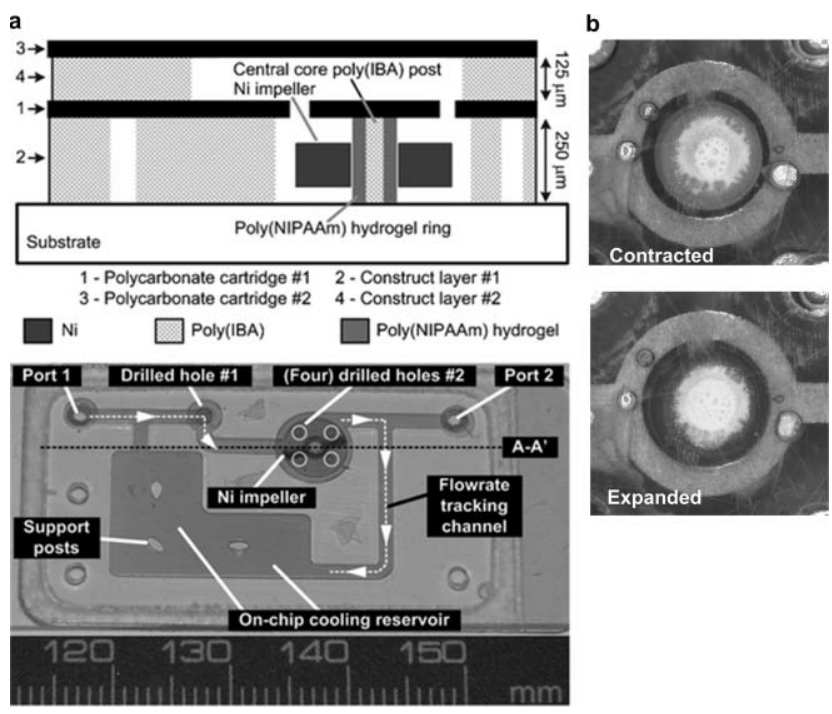

Fig. 7 An autonomously-triggered on-chip microfluidic cooling device. The device is driven by an external rotating magnetic stirrer. (a) Optical image and cross-section of the two-layer device. Holes drilled in cartridge \#1 allow fluid transfer between the two polymer construct layers. The temperature-responsive hydrogel ring acts similar to an automotive clutch by expanding and contracting in volume, and subsequently controlling the rotational status of the Ni impeller. The dashed white line and arrows show the partial pathway of the fluid as it is pumped and recirculated through the microchannels. The cooling reservoir is at room temperature. When local temperatures are above $32{ }^{\circ} \mathrm{C}$, the hydrogel contracts; the Ni impeller rotates, and pumps and recirculates cooler water. When local temperatures drop below $32{ }^{\circ} \mathrm{C}$, the hydrogel expands, thus the Ni impeller stops rotating. (b) The contracted (top) and expanded (bottom) hydrogel axle. Images reproduced from ref. 62 .

Many emerging lab-on-a-chip systems and biological microfluidics, such as PCR devices and DNA amplification, often require thermal management and necessitate multiple operation-temperature setpoints. Cooling, which can be facilitated by technologies such as air-cycling, and pumps, generally requires feedback sensors and control systems. Researchers used photopolymerizable polymers to fabricate a two-layer autonomous microfluidic cooling device in which a cooling liquid is kept circulating by a small nickel impeller driven by an external rotating magnetic stirrer (Fig. 7). ${ }^{62}$ A ring made of poly(NIPAAM) temperature-responsive hydrogel surrounding the impeller worked as a clutch as described above; it expanded and stopped the rotation of the impeller whenever the temperature drops below a given critical value, which could be tailored in advance (during hydrogel synthesis) according to the requirements of the chip in use. The device therefore provided autonomous control of the temperature by cooling rather than heating. When local temperatures rose above the defined temperature, the impeller autonomously responded by pumping and recirculating cooler water, until the local temperature decreased to a designed lower temperature. Active and adaptive microfluidic cooling pump arrays with different onset operation temperatures can be designed to fit microfluidic applications where on-chip multi-temperature management is necessary. 
Optical lenses are components of most optical systems, such as microscopes, telescopes, cameras, binoculars and magnifying glasses. In traditional man-made optical systems, a lens has a fixed focal length, and focusing of an object is achieved by physical displacement of the lens. In the human eye, on the other hand, objects at different distances are focused by adjusting the shape of the lens with the help of ciliary muscles. Jiang and his colleagues constructed autonomous liquid microlenses that could autonomously adapt to their local environment, where a stimulus-responsive hydrogel mimicking a muscle caused a change of the shape of the lens, i.e. the alteration of the focal length (Fig. 8(a-f)). ${ }^{63}$ The central component of the microfluidic chip was a hydrogel ring that was sandwiched between a glass plate and an aperture slip, and filled with water. The microlens was realized via a fluid interface between water and oil pinned at an aperture. The focal length of the lens was adjusted by changing the curvature of the meniscus. This was achieved by exposing the hydrogel ring to a stimulus causing the hydrogel to expand or shrink. Hence, the volume inside the hydrogel ring increased or
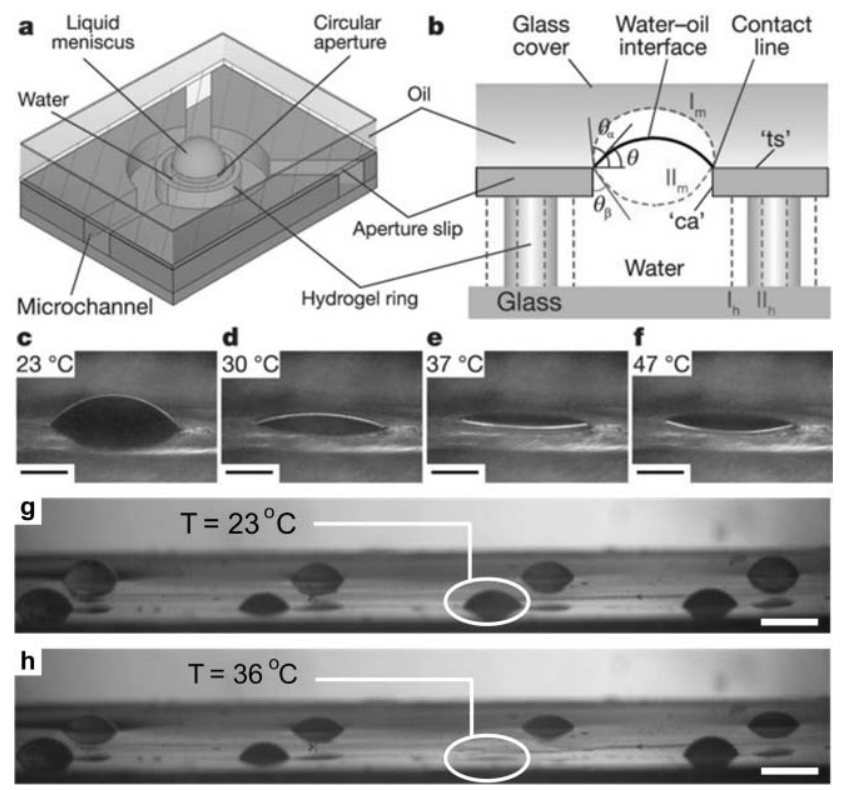

Fig. 8 Smart liquid microlenses. (a) Schematic of a liquid microlens. The water-oil interface forms the liquid microlens. The microchannels allow the flow of fluids to the microlens structure. (b) Smart variablefocus mechanism. A water-oil interface is pinned along a hydrophilichydrophobic contact line at an aperture. The hydrogel ring (tan), which wraps around a water reservoir and can expand (I, blue) or contract (II, red) in response to an external stimulus, thus propelling a water-oil surface upward (I) or downward (II). Because the refractive indices of the two liquids are different, the result is a variable-focus lens. (c-f) The shape of the liquid microlens varies with local environmental temperature using a temperature-sensitive hydrogel. Scale bars represent $1.0 \mathrm{~mm}$. $(\mathrm{g}-\mathrm{h})$ A liquid microlens array $(2 \times 4)$ where each lens can be individually controlled. All microlens elements use a temperature-responsive hydrogel with a lower critical solution temperature of $32{ }^{\circ} \mathrm{C}$, and are initially maintained at $23{ }^{\circ} \mathrm{C}$. When one element is heated to $36{ }^{\circ} \mathrm{C}$, it bows downward. Scale bars represent $1.0 \mathrm{~mm}$. (a-f) are reproduced from ref. 63. (g-h) are reproduced from ref. 64 with permission (copyright 2007, WILEY-VCH Verlag $\mathrm{GmbH} \&$ Co. KgaA).

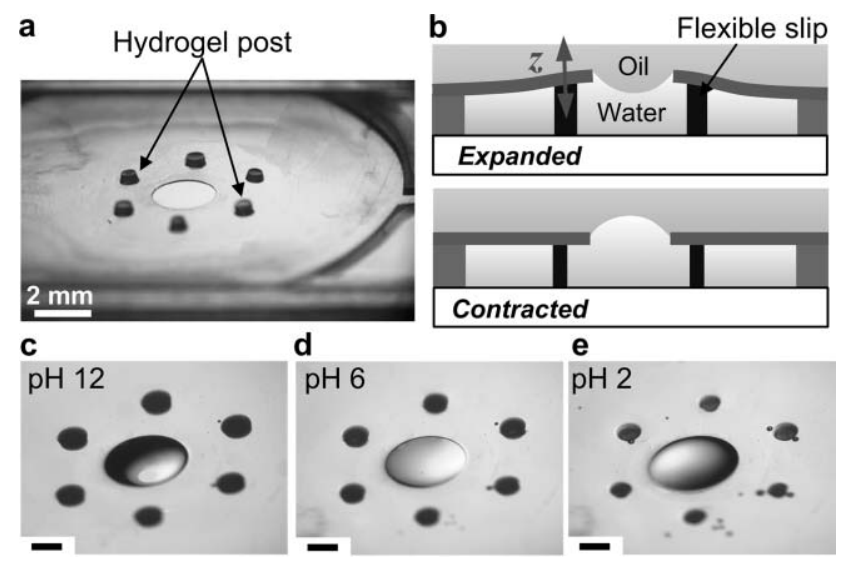

Fig. 9 A liquid microlens using vertical expansion and contraction of hydrogel. (a) A set of posts made of pH-responsive hydrogel is constructed in a microfluidic chamber. A circular aperture is formed in a flexible polymer slip. (b) The volume changes of hydrogel posts cause a flexible aperture slip to bend in the $z$-direction. The pinned water-oil interface shifts downward and upward, thus tuning the focal length of the microlens. (c-e) Shapes of liquid microlenses when varying $\mathrm{pH}$. Initially the hydrogel posts are at the expanded state ( $\mathrm{pH} 12)$. As low $\mathrm{pH}$ buffer ( $\mathrm{pH}$ 2) flows into the microfluidic chamber, the hydrogel posts contract, and the flexible slip bends back to press the liquid meniscus to bulge upward. Scale bars represent $1 \mathrm{~mm}$. Images reprinted with permission from ref. 65 (Copyright 2006, American Institute of Physics).

decreased, changing the pressure difference across the wateroil interface and thus, the geometry of the meniscus. The principle of the microlens was demonstrated with a poly(NIPAAM) temperature-sensitive hydrogel that expands at low temperatures and contracts at high temperature. For low temperature, the liquid meniscus grew, i.e. the microlens was divergent and varied the focal length from several $\mathrm{mm}$ to infinity. The concept was also proven utilizing a $\mathrm{pH}$-sensitive hydrogel. Also, temperature-responsive microlens arrays based on the same sensing and actuation mechanism were developed (Fig. 8(g-h)). ${ }^{64}$

The above concept of autonomous liquid microlens was implemented by harnessing the horizontal expansion and contraction of the hydrogel ring that separated lens liquid from buffer solution carrying stimuli information in the microfluidic channel. Another scheme that took advantage of the vertical expansion and contraction of multiple hydrogel posts was demonstrated (Fig. 9). ${ }^{65}$ This scheme can diversify the lens design to meet specific needs and is potentially beneficial for fast response time and mechanical robustness of the liquid microlens.

\section{Challenges}

Stimulus-responsive hydrogels have great potential for autonomous microfluidics, but there still exist a number of challenges. For example, the response time of hydrogels is still at disappointing time scales at this moment for many practical applications of the hydrogel-based devices. The dependence of the response time $T$, required for the volumetric change, on the size of the hydrogel is expressed as $T \approx L^{2} / D$, where $L$ is the smallest dimension of the hydrogel, and $D$ is 
the collective diffusion constant of the gel network. ${ }^{41}$ This equation indicates that a small size of hydrogel would give a faster response. For this purpose, one straightforward way is to downsize the hydrogel structures by using various microfabrication techniques. Increasing the porosity of hydrogel network has also been used to shorten the response time. ${ }^{66}$ Both methods, however, may degrade the mechanical properties of hydrogel structures, causing poor robustness of the devices. Therefore, how to realize a device with fast response while maintaining its mechanical robustness becomes critical. Further, key physical parameters such as the diffusion coefficients of ions, thermal diffusivities, and specific heats for various hydrogels need to be thoroughly characterized for better understanding and estimation of the volumetric change and response time.

\section{Conclusion}

The concept of microfluidic 'lab on a chip' has triggered tremendous effort in miniaturizing laboratory components that have the potential to integrate biology, chemistry, and medicine (often understood and applied at the macroscale) to be effectively applied at the microscale. The microscale offers numerous advantages such as dominance of surface tension, laminar flow, and diffusion that can be leveraged elegantly to design innovative microfluidic systems. Furthermore, it promises to exploit intelligent materials to achieve new functionalities and simplify the system structures. By incorporating stimuli-responsive hydrogels into microfluidics, advantages of autonomous microfluidics are compelling when simplicity, functionality, and intelligence are considered. Since chemical groups define the type of stimulus to which the hydrogel responds, and the physical properties determine the response time, hydrogels can be tuned to the desired application with a wide range of chemical and physical properties. This can greatly diversify the sensing and actuation mechanisms for autonomous microfluidics.

\section{Acknowledgements}

This work was supported by the U.S. Department of Homeland Security, through a grant awarded to the National Center for Food Protection and Defense at the University of Minnesota (Grant number N-00014-04-1-0659). Hongrui Jiang also thanks 3M Non-tenured Faculty Award for support. The authors thank Professor David J. Beebe and Chi-Wei Lo (University of Wisconsin-Madison) for their help in preparing the manuscript. The authors would like to express their sincere regrets to all researchers whose relevant work could not be discussed and cited in this article due to limited space.

\section{References}

1 G. M. Whitesides, "The origins and the future of microfluidics", Nature, 2006, 442, 368-373.

2 T. M. Squires and S. R. Quake, "Microfluidics: fluid physics at the nanoliter scale", Rev. Mod. Phys., 2005, 77, 977-1026.

3 D. Janasek, J. Franzke and A. Manz, "Scaling and the design of miniaturized chemical-analysis systems", Nature, 2006, 442, 374-380.
4 D. Psaltis, S. R. Quake and C. Yang, "Developing optofluidic technology through the fusion of microfluidics and optics", Nature, 2006, 442, 381-386.

5 P. Yager, T. Edwards, E. Fu, K. Helton, K. Nelson, M. R. Tam and B. H. Weigl, "Microfluidic diagnostic technologies for global public health", Nature, 2006, 442, 412-418.

6 J. El-Ali, P. K. Sorger and K. F. Jensen, "Cells on chips", Nature, 2006, 442, 403-411.

7 G. H. W. Sanders and A. Manz, "Chip-based microsystems for genomic and proteomic analysis", Trends Anal. Chem., 2000, 19, 364-378.

8 C. Monat, P. Domachuk and B. J. Eggleton, "Integrated optofluidics: a new river of light", Nat. Photonics, 2007, 1, 106-114.

9 P. Vadgama and P. W. Crump, "Biosensors recent trends - a review", Analyst, 1992, 117, 1657-1670.

10 M. L. Chabinyc, D. T. Chiu, J. C. McDonald, A. D. Stroock, J. F. Christian, A. M. Karger and G. M. Whitesides, "An integrated fluorescence detection system in poly(dimethylsiloxane) for microfluidic applications", Anal. Chem., 2001, 73, 4491-4498.

11 D. A. Czaplewski, B. R. Ilic, M. Zalalutdinov, W. L. Olbricht, A. T. Zehnder, H. G. Craighead and T. A. Michalske, "A micromechanical flow sensor for microfluidic applications", J. Microelectromech. Syst., 2004, 13, 576-585.

12 H. Berthou and C. K. Jorgensen, "Optical-fiber temperature sensor based on upconversion-excited fluorescence", Opt. Lett., 1990, 15, $1100-1102$.

13 W. P. Eaton and J. H. Smith, "Micromachined pressure sensors: review and recent developments", Smart Mater. Struct., 1997, 6, $530-539$.

$14 \mathrm{~K}$. W. Oh and C. H. Ahn, "A review of microvalves", J. Micromech. Microeng., 2006, 16, R13-R39.

15 P. Woias, "Micropumps - past, progress and future prospects", Sens. Actuators, B, 2005, 105, 28-38.

16 N. T. Nguyen and Z. G. Wu, "Micromixers - a review", J. Micromech. Microeng., 2005, 15, R1-R16.

17 L. Zhu, Q. Zhang, H. H. Feng, S. Ang, F. S. Chauc and W. T. Liu, "Filter-based microfluidic device as a platform for immunofluorescent assay of microbial cells", Lab Chip, 2004, 4, 337-341.

18 P. J. A. Sazio, A. Amezcua-Correa, C. E. Finlayson, J. R. Hayes, T. J. Scheidemantel, N. F. Baril, B. R. Jackson, D. J. Won, F. Zhang, E. R. Margine, V. Gopalan, V. H. Crespi and J. V. Badding, "Microstructured optical fibers as high-pressure microfluidic reactors", Science, 2006, 311, 1583-1586.

19 M. U. Kopp, A. J. de Mello and A. Manz, "Chemical amplification: continuous-flow PCR on a chip", Science, 1998, 280, 1046-1048.

20 G. Maltezos, A. Rajagopal and A. Scherer, "Evaporative cooling in microfluidic channels", Appl. Phys. Lett., 2006, 89, 074107 .

21 D. J. Beebe, J. Moore, J. Bauer, Q. Yu, R. H. Liu, C. Devadoss and B. H. Jo, "Functional hydrogel structures for autonomous flow control inside microfluidic channels", Nature, 2000, 404, $588-590$.

22 A. S. Hoffman, "Hydrogels for biomedical applications", $A d v$. Drug Delivery Rev., 2002, 54, 3-12.

23 W. Khun, B. Hargitay, A. Katchalsky and H. Eisenberg, "Reversible dilation and contraction by changing the state of ionization of high-polymer acid networks", Nature, 1950, 165, 514-516.

24 K. Y. Lee and D. J. Mooney, "Hydrogels for tissue engineering", Chem. Rev., 2001, 101, 1869-1879.

25 T. Tanaka, D. Fillmore, S. Sun, I. Nishio, G. Swislow and A. Shah, "Phase transitions in ionic gels", Phys. Rev. Lett., 1980, 45, $1636-1639$

26 K. Kataoka, H. Miyazaki, M. Bunya, T. Okano and Y. Sakurai, "Totally synthetic polymer gels responding to external glucose concentration: their preparation and application to on-off regulation of insulin release", J. Am. Chem. Soc., 1998, 120, 12694-12695.

27 T. Miyata, N. Asami and T. Uragami, "A reversible antigenresponsive hydrogel", Nature, 1999, 399, 766-796.

28 J. Kim, S. Nayak and L. A. Lyon, "Bioresponsive hydrogel microlenses", J. Am. Chem. Soc., 2005, 127, 9588-9592. 
29 J. Hoffman, M. Plotner, D. Kuckling and W. Fischer, "Photopatterning of thermally sensitive hydrogels useful for microactuators", Sens. Actuators, B, 1999, 77, 139-144.

30 A. Suzuki and T. Tanaka, "Phase transition in polymer gels induced by visible light", Nature, 1990, 346, 345-347.

31 T. Tanaka, S. Sun, I. Nishio and S. Ueno-Nishio, "Collapse of gels in an electric field", Science, 1982, 218, 467-469.

32 J. Moorthy and D. J. Beebe, "Organic and biomimetic designs for microfluidic systems - new strategies offer a flexible approach to designing microscale devices", Anal. Chem., 2003, 75, 292A-301A.

33 A. Richter, D. Kuckling, S. Howitz, T. Gehring and K. F. Arndt, "Electronically controllable microvalves based on smart hydrogels: magnitudes and potential applications", J. Microelectromech. Syst., 2003, 12, 748-753.

34 D. J. Beebe, J. S. Moore, Q. Yu, R. H. Liu, M. L. Kraft, B. H. Jo and C. Devadoss, "Microfluidic tectonics: a comprehensive construction platform for microfluidic systems", Proc. Natl. Acad. Sci. U. S. A., 2000, 97, 13488-13493.

35 M. Lei, Y. D. Gu, A. Baldi, R. A. Siegel and B. Ziaie, "Highresolution technique for fabricating environmentally sensitive hydrogel microstructures", Langmuir, 2004, 20, 8947-8951.

36 A. K. Agarwal, D. J. Beebe and H. Jiang, "Integration of polymer and metal microstructures using liquid-phase photopolymerization", J. Micromech. Microeng., 2006, 16, 332-340.

37 Y. Qiu and K. Park, "Environment-sensitive hydrogels for drug delivery", Adv. Drug Delivery Rev., 2001, 53, 321-339.

38 A. S. Hoffman, "Hydrogels for biomedical applications", Ann. N. Y. Acad. Sci., 2001, 944, 62-73.

39 A. M. Mathur, S. K. Moorjani and A. B. Scranton, "Methods for synthesis of hydrogel networks: a review", Polym. Rev., 1996, 36, 405-430.

40 Y. Osada, J. P. Gong and Y. Tanaka, "Polymer gels", J. Macromol. Sci., Polym. Rev., 2004, C44, 87-112.

41 H. Suzuki, "Stimulus-responsive gels: promising materials for the construction of micro actuators and sensors", J. Intell. Mater. Syst. Struct., 2006, 17, 1091-1097.

42 R. H. Liu, Q. Yu and D. J. Beebe, "Fabrication and characterization of hydrogel-based microvalves", J. Microelectromech. Syst., 2001, 11, 45-53.

43 Q. Yu, J. M. Bauer, J. S. Moore and D. J. Beebe, "Responsive biomimetic hydrogel valve for microfluidics", Appl. Phys. Lett., 2001, 78, 2589-2591.

44 D. Kim and D. J. Beebe, "A bi-polymer micro one-way valve", Sens. Actuators, A, 2007, 136, 426-433.

45 Q. Z. Luo, S. Mutlu, Y. B. Gianchandani, F. Svec and J. M. J. Frechet, "Monolithic valves for microfluidic chips based on thermoresponsive polymer gels", Electrophoresis, 2003, 24, 3694-3702.

46 A. Baldi, Y. Gu, P. Loftness, R. A. Siegel and B. Ziaie, "A hydrogel-actuated environmentally-sensitive microvalve for active flow control", J. Microelectromech. Syst., 2003, 12, 613-621.

$47 \mathrm{~S}$. Bohm, W. Olthuis and P. Bergveld, "An integrated micromachined electrochemical pump and dosing system", Biomed. Microdevices, 1999, 1, 121-129.
48 C. Laritz and L. Pagel, "A microfluidic pH-regulation system based on printed circuit board technology", Sens. Actuators, A, 2000, 84, 230-235.

49 D. T. Eddington, R. H. Liu, D. J. Beebe and J. S. Moore, "An organic self-regulating microfluidic system”, Lab Chip, 2001, 1, 96-99.

50 D. T. Eddington and D. J. Beebe, "Flow control with hydrogels, flow control with hydrogels", Adv. Drug Delivery Rev., 2004, 56, 199-210.

51 X. Cao, S. Lai and L. J. Lee, "Design of a self-regulated drug delivery device", Biomed. Microdevices, 2001, 3, 109-118.

52 R. H. Liu, M. A. Stremler, K. V. Sharp, M. G. Olsen, J. G. Santiago, R. J. Adrian, H. Aref and D. J. Beebe, "Passive mixing in a three-dimensional serpentine microchannel", J. Microelectromech. Syst., 2000, 9, 190-197.

53 M. Barbic, J. J. Mock, A. P. Gray and S. Schultz, "Electromagnetic micromotor for microfluidics applications", Appl. Phys. Lett., 2001, 79, 1399-1401.

54 A. D. Stroock, S. K. W. Dertinger, A. Ajdari, I. Mezic, H. A. Stone and G. M. Whitesides, Chaotic mixer for micro channels, Science, 2002, 295, 647-651.

55 L.-H. Lu, K. S. Ryu and C. Liu, "A magnetic microstirrer and array for microfluidic mixing", J. Microelectromech. Syst., 2002, 11, 462-469.

56 K. S. Ryu, K. Shaikh, E. Goluch, Z. Fan and C. Liu, "Micro magnetic stir-bar mixer integrated with parylene microfluidic channels", Lab Chip, 2004, 4, 608-613.

57 K.-S. Yun, I.-J. Cho, J.-U. Bu, C.-J. Kim and E. Yoon, “A surfacetension driven micropump for low-voltage and low-power operations", J. Microelectromech. Syst., 2002, 11, 454-461.

58 J. W. Munyan, H. V. Fuentes, M. Draper, R. T. Kelly and A. T. Woolley, "Electrically actuated, pressure-driven microfluidic pumps", Lab Chip, 2003, 3, 217-220.

59 J. Atencia and D. J. Beebe, "Magnetically-driven biomimetic micropumping using vortices", Lab Chip, 2004, 4, 598-602.

60 N.-T. Nguyen and T.-Q. Truong, "A fully polymeric micropump with piezoelectric actuator", Sens. Actuators, B, 2004, 97, 137-143.

61 A. K. Agarwal, S. S. Sridharamurthy, D. J. Beebe and H. Jiang, "Autonomous micromixers and micropumps", J. Microelectromech. Syst., 2005, 14, 1409-1421.

62 A. K. Agarwal, L. Dong, D. J. Beebe and H. Jiang, "Autonomously-triggered microfluidic cooling using thermoresponsive hydrogels", Lab Chip, 2007, 7, 310-315.

63 L. Dong, A. K. Agarwal, D. J. Beebe and H. Jiang, "Adaptive liquid microlenses activated by stimuli-responsive hydrogels", Nature, 2006, 442, 551-554.

64 L. Dong, A. K. Agarwal, D. J. Beebe and H. Jiang, "Variablefocus liquid microlenses and microlens arrays actuated by thermoresponsive hydrogels", Adv. Mater., 2007, 19, 401-405.

65 L. Dong and H. Jiang, "pH-adaptive microlenses using pinned liquid-liquid interfaces actuated by $\mathrm{pH}$-responsive hydrogel", Appl. Phys. Lett., 2006, 89, 211120.

66 B. Zhao and J. S. Moore, "Fast pH- and ionic strength-responsive hydrogels in microchannels", Langmuir, 2001, 17, 4758-4763. 